\title{
-NOTES-
}

\section{A SECOND NOTE ON COMPRESSIBLE FLOW ABOUT BODIES OF REVOLUTION*}

\author{
By W. R. SEARS (Cornell University)
}

In a recent Note ${ }^{1}$ the present author discussed the application of the linear-perturbation theory to the subsonic flow of a compressible fluid past a slender bod of revolution. It was shown that the several variants of the theory $y^{2,3,4,5,6}$ lead to inconsistent results when applied, for example, to the flow about ellipsoids of revolution, and it was stated that this ambiguity results from applying the theory to a case that is essentially nonlinear. It was implied that all the variants are equally valid so far as the first-order theory is concerned.

In the present Note it will be shown that the various procedures are not, in fact, equally acceptable, and that only one of them, in which the boundary condition is properly satisfied, can be considered to be correct, even to the first order.

The boundary-value problem involved is the following:

$$
\left.\begin{array}{c}
\beta^{2} \varphi_{x x}+(1 / r)\left(r \varphi_{r}\right)_{r}=0, \\
\frac{\varphi_{r}}{U+\varphi_{x}}=\frac{d R}{d x}=R^{\prime}(x), \text { say, when } r=R(x), \\
\varphi \rightarrow 0 \text { as either }|x| \rightarrow \infty \text { or } r \rightarrow \infty .
\end{array}\right\}
$$

Here $\phi=U x+\varphi$ is the velocity potential, $x$ and $r$ are the usual cylindrical coordinates, and $\beta^{2}=1-M^{2}$, a constant defining the Mach number of the undisturbed flow. The body of revolution is defined by the function $R(x)$. The perturbation velocities $\varphi_{x}$ and $\varphi_{r}$ have been neglected in comparison with the free-stream velocity $U$; similarly, $\varphi_{x}$ can be neglected in the first boundary condition in (1).

It is easily ascertained that solutions I, II, and III of the earlier Note ${ }^{1}$ do not accurately satisfy this boundary condition. These solutions were derived from the incompressible-flow solution $f(x, r)$, which satisfies the following conditions:

$$
\left.\begin{array}{c}
f_{x x}+(1 / r)\left(r f_{r}\right)_{r}=0, \\
f_{r}(x, S)=U \cdot d S / d x=U S^{\prime}(x), \\
f \rightarrow 0 \text { say; } \\
f \rightarrow \text { as either }|x| \rightarrow \infty \quad \text { or } \quad r \rightarrow \infty
\end{array}\right\}
$$

* Received Sept. 18, 1946.

${ }^{1}$ W. R. Sears, On compressible flow about bodies of revolution, Quart. Appl. Math. 4, 191-193 (1946).

2 S. Goldstein and A. D. Young, The linear perturbation theory of compressible flow with applications to wind-tunnel interference, British Aero. Res. Com. Reports and Memoranda No. 1909 (1943).

${ }^{3}$ H. S. Tsien and L. Lees, The Glauert-Prandtl approximation for subsonic flow of a compressible fluid, J. Aero. Sci. 12, 173-187, 202 (1945).

$4 \mathrm{H}$. W. Liepmann and A. Puckett, Introduction to the aerodynamics of compressible fluids, John Wiley and Sons, New York, 1946.

' R. Sauer, Theoretische Einfïhrung in die Gasdynamik, Springer, Berlin, 1943. Reprinted by Edwards Bros., Inc., Ann Arbor, 1945.

- B. Göthert, Ebene und räumliche Strömung bei hohen Unterschallgeschwindigkeiten, Lilienthal Gesellschaft f. Luftfahrtforschung, Bericht 127, 97-101 (1940). 
In each solution, the body $R(x)$ is supposed to be related to the different body $S(x)$ in such a manner as to satisfy the boundary conditions. The various solutions are tabulated below, and in each case is stated the boundary condition that is actually satisfied:

$$
\begin{aligned}
\varphi(x, r) & =\frac{1}{\beta} f(x, \beta r), \\
\varphi_{r}(x, S / \beta) & =U S^{\prime}(x) ; \\
\varphi(x, r) & =f(x, \beta r), \\
\varphi_{r}(x, S / \beta) & =\beta U S^{\prime}(x) ; \\
\varphi(x, r) & =f(x / \beta, r), \\
\varphi_{r}(x, S) & =U S^{\prime}(x / \beta) ;
\end{aligned}
$$

II

III

IV

$$
\varphi(x, r)=\lambda f(x, \beta r),
$$

$$
\varphi_{r}(x, S / \beta)=\lambda \beta U S^{\prime}(x) ;
$$

$$
\begin{aligned}
\phi=\beta^{2} U x+f(x, \beta r) & =\beta^{2} U x+\varphi(x, r), \text { say; } \\
\varphi_{r}(x, S / \beta) & =U \beta S^{\prime}(x)=\left(\beta^{2} U\right) S^{\prime}(x) / \beta .
\end{aligned}
$$

It is seen that only Method $\mathrm{V}$, and Method IV with $\lambda=1 / \beta^{2}$, satisfy in the $x, r$ space the correct (linearized) boundary condition for flow about a solid body; i.e., that the stream surface has the same slope as the body at the surface of the body. Thus the ambiguity mentioned in the Note is eliminated and the formula for the maximum superstream velocity ratio on the surface becomes unique:

$$
\frac{\phi_{x}-U}{U}=\frac{1}{\beta^{2}} F(\beta n),
$$

where $n$ is the ratio of maximum diameter to length for the particular family of bodies under consideration, and $F(n)$ is the maximum superstream velocity ratio in incompressible flow.

The reason for the confusion mentioned in the earlier Note, and undoubtedly contributed to by that Note, is not the nonlinear character of $F(n)$, but the fact that appreciable errors are introduced by use of the approximate boundary conditions shown in I-III above, in the three-dimensional case. In the analogous two-dimensional case, to be sure, the boundary values of the normal velocity component can be satisfied at an approximate location-it is customary to fix them along a straight line, for convenience - without introduction of any error in a first-order theory. Thus the variants, which differ only in the location where the slope is fixed, all produce the same result. This is not true, in general, in the three-dimensional case. This fact was overlooked by various writers ${ }^{2,3,5}$ and by the present author in the earlier Note. ${ }^{1}$

The essential difference between the plane case and that of axial symmetry is understood by consideration of the distribution of singularities, along the axis, that is needed to produce the required flow. In the plane case, both the normal and the axial (downstream) velocity components $u$ and $v$, are constant, to the first order, through a small distance each side of the surface of discontinuity; hence an approximation to the position at which the boundary values of $v$ are fixed does not affect the 
strength of singularities nor the resulting values of $u$. In the axially-symmetrical case, on the other hand, for any source-sink distribution along the axis, $\phi_{r}$ varies as $1 / r$ while $\phi_{x}$ is nearly constant, for small $r$. Thus an approximation to the radius at which the boundary values of $\phi_{r}$ occur causes appreciable errors in $\phi_{x}{ }^{7}$

In conclusion, it now appears, after further study, that the conclusions of the earlier Note are in error, and that Method V, or its equivalent, is the only correct one for the case of axially-symmetric flow. Method V is that employed by Göthert, ${ }^{6}$ and it must be agreed that his objections to the conventional procedure (Method II), which the author previously termed "fancied," are, in fact, valid. ${ }^{8}$

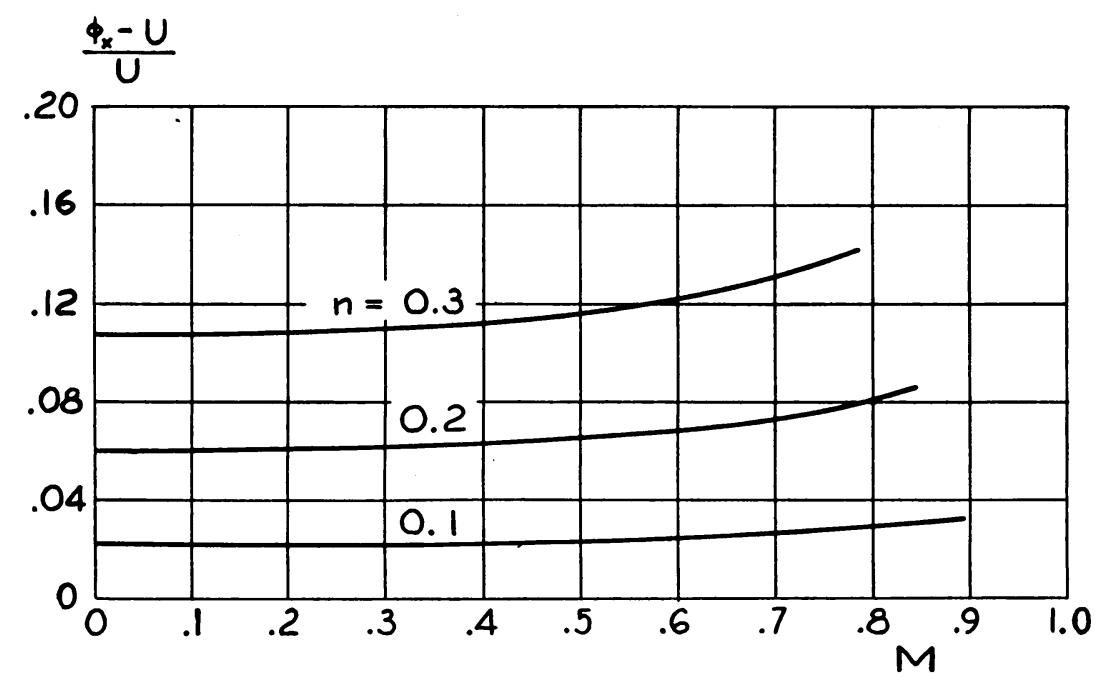

FIG. 1. The maximum superstream velocity ratio for ellipsoids of revolution in compressible flow. $M$ is the free-stream Mach number and $n$ is the ratio of maximum diameter to length of the ellipsoid.

For the particular case of ellipsoids of revolution, the results of Method V are shown in Figure 1. It should particularly be noted that the correct velocity-ratio formula, equation (3), does not permit a universal velocity-correction curve to be drawn as function of the Mach number, as the result of Method I would, for example, and as in the two-dimensional case. The effect of increasing $M$ is seen to depend intimately upon $n$ and upon the nature of $F(n)$; hence the curves of Figure 1 cannot be expected to apply to bodies other than the ellipsoids of revolution.

${ }^{7}$ In the paper of Goldstein and Young (loc. cit.) this approximation is introduced in equation (21) for example, when $y$ is replaced by $h$ in integrating to determine $y-h$.

${ }^{8}$ Note added in proof: In a report recently released [L. Lees, $A$ discussion of the application of the Prandtl-Glauert method to subsonic compressible flow over a slender body of revolution, N.A.C.A.Techn. Note No. 1127], one of the authors mentioned here has reconsidered the problem and has arrived at conclus:ons in complete agreement with those of the present note. 